\title{
Simulated Annealing Algorithm Based on Gauss Distribution
}

\author{
Sang Jie, Zhan Hu, Song Chun-lin* \\ School of Internet of Things (IoT) Engineering, Jiangnan University, Wuxi, China
}

\section{Email address:}

sarlly2016@hotmail.com (Sang Jie), zhanhujiangnan@163.com (Zhan Hu), songchunlin@jiangnan.edu.cn (Song Chun-lin)

${ }^{*}$ Corresponding author

\section{To cite this article:}

Sang Jie, Zhan Hu, Song Chun-lin. Simulated Annealing Algorithm Based on Gauss Distribution. Science Discovery. Vol. 4, No. 1, 2016, pp. 52-55. doi: 10.11648/j.sd.20160401.19

Received: April 7, 2016; Accepted: April 11, 2016; Published: April 18, 2016

\begin{abstract}
This paper first describes in a brief manner the principle, the building blocks and the realization of the classical simulated annealing (SA) algorithm. Its weakness is also discussed and an enhanced SA algorithm is then proposed. The new algorithm tackles the global optimization and the local optimization processes separately. With an enhanced non-uniform mutation method, the global search range is expanded, which also leads to an improved local optimal solution. Finally, this paper uses a real-world optimization problem to contrast the conventional and the enhanced SA algorithms, and demonstrates the superiority of the newly proposed technique.
\end{abstract}

Keywords: Simulated Annealing Algorithm, Combinatorial Optimization, Gauss Distribution, Metropolis Sampling, Global Optimization

\section{基于高斯分布的模拟退火算法及其应用}

\author{
桑捷, 展虎, 宋春霖 ${ }^{*}$ \\ 物联网工程学院, 江南大学, 无锡, 中国
}

\section{邮箱}

sar1ly2016@hotmail.com（桑捷），zhanhujiangnan@163.com（展虎），songchunlin@jiangnan.edu.cn（宋春霖）

\begin{abstract}
摘要：这篇文章介绍了传统模拟退火算法的原理、基本组成、实现方法, 并详细分析了它存在的不足, 提出了该算法 的改进措施。改进的模拟退火算法将全局优化和局部优化过程分开, 并采用了一个改进的非均匀变异的扰动模型, 扩 大了全局搜索范围, 提高了局部优化的效果。针对不同的温度区间, 采取不同的降温策略, 使得最优性在被保证的前 提下减少了计算量。最后针对一个实际的优化问题, 使用改进前后的两种算法进行实验, 实验证明改进后的模拟退火 算法的有效性。
\end{abstract}

关键词：模拟退火算法, 组合优化, 高斯分布, 蒙特卡洛, 全局优化

\section{1. 引言}

在自然科学、社会科学以及管理科学等领域存在着大 量的优化问题。特别是从 20 世纪 80 年代以来, 优化问题的
求解在VLSI生产调度、控制工程、图像处理等 [1] 工程领 域等的需求日益增加。

许多实际的优化问题都是非凸 (Non-convex) 的, 存在 较多的局部最优解。因此, 有效寻找非凸优化问题的全局 
最优解一直是研究热点之一, 如物流配送 [2]。人们提出 了两类方法用于寻找非凸问题的全局最优解: 一类是确定 性方法, 另一类是随机性方法。前者基于确定性的搜索策 略, 后者在搜索中引入了的随机扰动, 使得求解算法的适 用性变得更加广泛 [3]。

模拟退火算法（Simulated Annealing (SA) Algorithm）是一种基于随机搜索策略 [4]的方法, 该方法 是针对局部搜索算法进行改进 [5]而提出的, 可求解大规 模的非线性优化问题。其主要由解空间、目标函数和初始 解三部分组成。SA算法通过类比统计力学中的热平衡问题, 模拟固体退火过程, 搜索无约束优化问题的全局最优解, 在各种实际中有着广泛的运用。

\section{2. 模拟退火算法的原理}

\section{1. 模拟退火算法的提出}

SA算法的基本思想最早可以追溯到 1953年, 是由 Metropolis 等人在研究二维相变时提出的。1983 年 kirkpatrick [6] 等人成功地将其引入到优化领域中, 从此 $\mathrm{SA}$ 算法开始被大规模、广泛地应用到非线性优化求解的问 题中 [7]。

\section{2. 模拟退火算法的原理}

$\mathrm{SA}$ 算法综合了统计物理学知识和局部搜索技术, 是模 拟固体退火过程的全局优化算法, 与物理退火过程有很强 的相似性, 其类比关系如表1所示。SA算法在模拟固体退 火过程的自然机理的同时, 引入合适的随机扰动因子 [8], 在降温过程中不断优化参数值, 也以一定概率接收较差的 解。随着温度的逐渐降低, SA算法逐渐跳出全局搜索过程, 进入局部优化过程, 以提高解的精度。

表1 模拟退火算法与物理退火过程的类比关系。

\begin{tabular}{ll}
\hline 物理退火过程 & 模拟退火算法 \\
\hline 固体内部状态 & 解空间 \\
状态能量 & 解的质量 \\
温度 & 控制参数 \\
熔解过程 & 初始温度 \\
退火冷却过程 & 参数的扰动 \\
状态转移 & 解的变化 \\
能量最低状态 & 最优解 \\
\hline
\end{tabular}

SA算法一般包含了Metropolis抽样 [9] 及合适的降温 函数。Metropolis抽样的原理如下: 假定固体初始状态为 $i$, 能量表示为 $\mathrm{E}(\mathrm{i})$; 为当前状态随机选择一个微小变化 进行扰动, 得到一个新状态 $j$, 能量表示为 $E(j)$, 若 $E(j)<E(i)$, 则将当前状态更新为 $j$ 状态; 若 $E(j)>E(i)$, 则根据该固体处于该状态的概率

$$
\exp ((E(i)-E(j)) / T)
$$

决定是否接受相对 $\mathrm{i}$ 而言较差的解 $\mathrm{j}$ 为更新状态。在当 前温度下 $\mathrm{T}$, 该随机搜索过程多次进行, 以寻找优化问题
的较优解; 随后降低当前温度, 在新的温度下, 重复上述 Metropolis抽样过程。SA算法交替进行固定温度下的随机 搜索和退火 (降温) 过程, 实现对优化问题解的全局搜索 和局部搜索。

\section{3. 模拟退火算法的基本组成}

$\mathrm{SA}$ 算法一般包含如下的 “三函数两准则” :

(1) 新状态产生函数: 以一定的方式产生随机扰动, 令候选解可以遍及整个解空间; 它包括扰动产生方式和候 选解的概率分布。

(2) 状态接受函数：以一定的概率接受代价函数值得 偶然上升, 此概率函数称为接受函数 $[10]$ 。

(3) 退温函数：在考虑优化质量和效率的前提下，采 取不同的退温策略, 通常有快速退温、指数退温、对数退 温及直线退温四种策略。

(4) 抽样稳定准则: 用于接受更新状态的判决准则。

(5) 退火结束准则: 随着控制参数 $\mathrm{T}$ 的逐渐减小, 令 $\mathrm{T}$ 在达到某个低温时, 退出SA算法。

\section{4. 模拟退火算法的实现方法}

SA算法实现的具体步骤如下:

(1) 随机选择一个初始状态 $\mathrm{m} 0$, 计算相应的能量函数 值为E 0 , 并设初始温度为 $\mathrm{T} 0$, 设置 $\mathrm{i}=0$;

(2) 对当前模型进行扰动处理, 得到新状态 $m$, 并计算 扰动后的能量函数E1, 利用Metropolis 抽样准则确定是否 接受新状态 $m$;

(3) 在温度 $\mathrm{Ti}$ 下重复一定次数的步骤（2），并根据合 适的降温策略得到新的温度值 $\mathrm{T} i+1$;

(4) 检查温度是否满足终止准则, 若满足则输出最优 解, 若不满足则重复步骤（2）和（3）。

\section{5. 模拟退火算法的不足}

$\mathrm{SA}$ 算法是类比固体退火而得出的优化算法, 在每个温 度 Ti下, 经过重复Metropolis抽样准则, 都会得到满足玻 尔兹曼分布规律的函数值, 随着温度的逐渐下降, 参数分 布会逐渐趋向于全局最小能量状态的分布状态 [11]。但与 此同时, SA算法也会存在如下的一些不足之处:

(1) SA算法的全局最优解与计算时间存在着矛盾, SA 算法会以较高概率收玫于一个全局最优点, 但花费的时间 却并不理想。

(2) 全局优化与局部优化过程采取相同的降温策略和 扰动策略, 不能同时将两种优化效果最大化。

(3) 在产生优解的同时, 也有概率会保存差解, 传统 $\mathrm{SA}$ 算法没有储存各温度最优解的记忆器, 在一定概率上易 造成输出的最优解并不理想。

\section{3. 模拟退火算法的改进}

2.5节分析了SA算法算法中的不足之处，结合SA算法 在实际项目中的应用, 其采取的改进方法如下:

(1) 将SA算法的退火结束准则面向全局优化和局部优 化过程分开讨论, 此时, 如何选择退出全局优化进入局部 
优化的温度非常重要。此处将扰动因子和待求系数入同一 数量级时的温度 $\mathrm{Ti}$ 定为分界温度。

（2）借鉴遗传算法中的非均匀变异思想 [12]，用非均 匀的变异策略对当前参数状态进行扰动, 产生新的参数状 态 [13] :

在全局搜索过程中, 搜索范围广, 扰动因子往往不在 一个数量级上, 因此选取均匀分布的随机数来实现全局搜 索策略 [14]:

$$
\operatorname{Infection}(i)=r^{*}\left(T_{i} / N\right)^{\lambda}
$$

其中, $r$ 是 $(-1,1)$ 均匀分布的随机数, $T i$ 当前温度, $N$ 为最大循环次数, $\lambda$ 为非均匀性程度的常数, 此过程 $\lambda$ $=0.5$, 其扰动结果如图1所示。

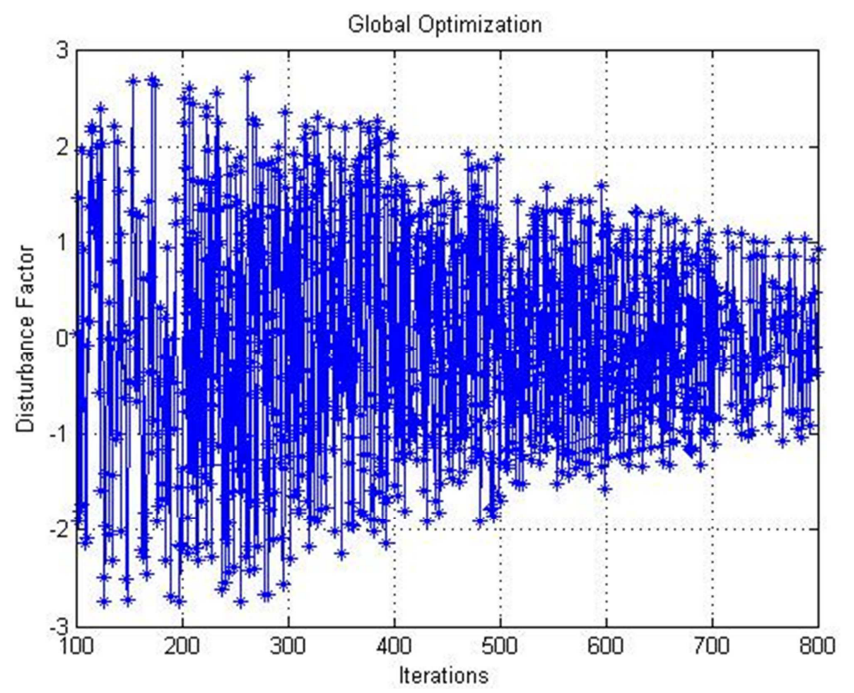

图1 全局扰动搜索分析图。

图1中 $\mathrm{x}$ 轴表示循环次数, $\mathrm{y}$ 轴表示全局搜索的扰动因 子。从图1发现此全局优化过程搜索范围广, 且有明显收 敛趋势。

在局部搜索过程中, 搜索区间减小, 进入对数据的深 度优化进程, 此时数据变化相对较小, 参数变化符合高斯 分布。此处在选取随机值扰动时, 为了提升产生有效扰动 的概率, 也能在小概率上发现全局搜索可能错过的最优解, 因此选取高斯分布的随机数来实现局部深度搜索:

$$
\text { Infection }(i)=R^{*}\left(T_{i} / N\right)^{\lambda}
$$

其中, $\mathrm{R}$ 是 $(-1,1)$ 高斯分布的随机数, 在此过程中 $\lambda$ $=2.5$, 其扰动结果如图2所示。

图2中x轴表示循环次数, $\mathrm{y}$ 轴表示局部搜索的扰动因 子。从图2发现局部优化过程搜索范围缩小, 优化效果更 精确, 最终实现局部收玫。

(3) 在不同优化状态下, 采取不同的降温策略, 此 处在全局优化中采用快速降温策略, 增加内循环次数; 在 局部优化中采用指数降温策略, 保持内循环次数不变。

通过这样的改进策略，使SA算法能将不同的优化过程 效果最大化, 且算法可应用型被提高。在优化相同数量的 数据时, 改进的SA算法比传统 $\mathrm{SA}$ 算法更加稳定, 而且产生
最优解的时间在一定程度上被缩短。第四部分通过对养猪 场数据的对比, 来比较分析改进的SA算法算法和传统 SA 算法算法。

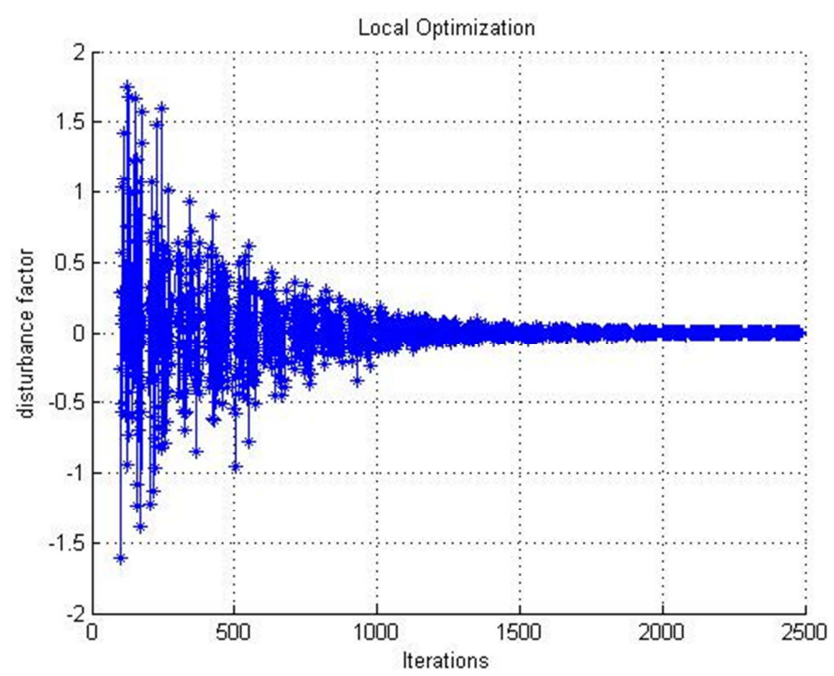

图2 局部扰动搜索分析图。

\section{4. 改进的模拟退火算法实验}

通过对养殖户负面行为处理病死猪的路径研究, 提取 养猪场规模产值关于资金、劳动力、养殖场面积、环境要 素的 $5 \times 153$ 维的数据, 如图 3 所示:

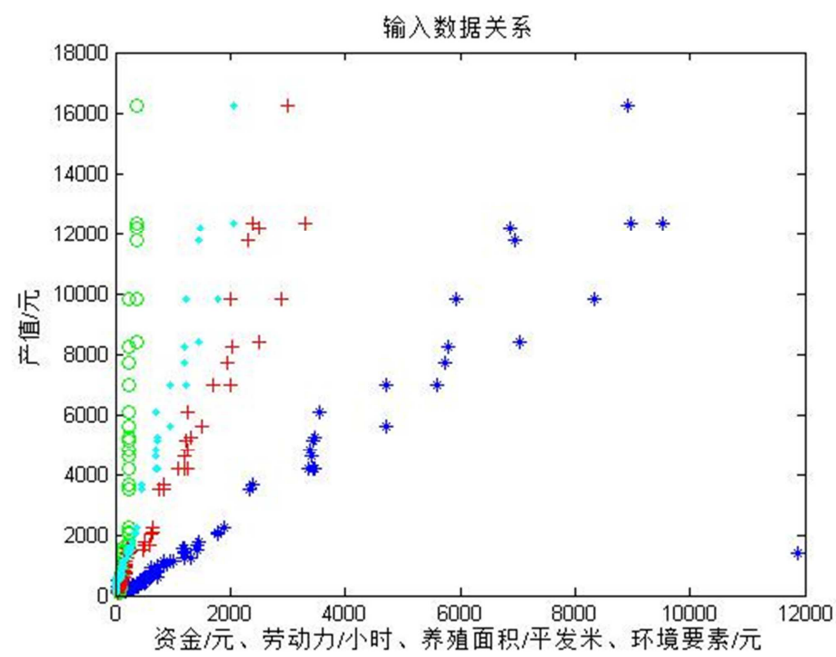

图3 养猪场产值数据关系图。

结合Cobb-Douglas生产函数模型 $[15]$ :

$$
Y=A^{*} X_{1} * \beta_{1} * X_{2} * \beta_{2} * X_{3} * \beta_{3} * X_{4} * \beta_{4}
$$

其中, $A$ 代表综合技术水平， $\beta 1 、 \beta 2 、 \beta 3 、 \beta 4$ 都 是弹性系数, Y表示产值, X表示自变量。在调用相同初值 的前提下, 采用改进前后的SA算法对数据进行优化处理的 实验分析, 调取产值关于环境要素在改进的SA算法下拟合 结果如图4所示: 


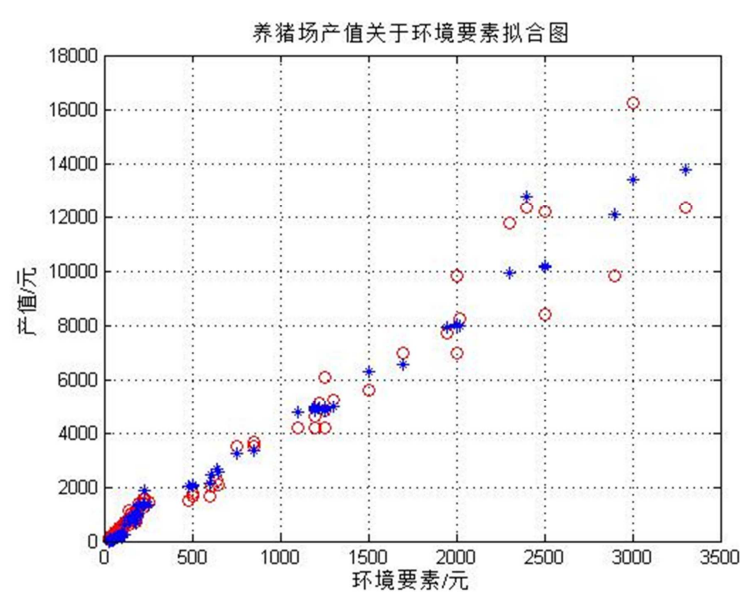

图4 改进的SA算法优化拟合图。

通过各系数初值得出的拟合度是 $82.26 \%$, 经过改进后 的SA算法的拟合度被稳定在 $96.85 \%$ 左右, 比传统的SA算法 稳定, 减小了产生不理想最优解的概率, 同时时间也被缩 短了 $0.5 \mathrm{~s}$ 左右。实验证明, 改进的 SA算法合理高效。

\section{5. 结论}

本文首先从原理、基本组成、实现方法三个方面介绍 了SA算法。传统的SA算法从本质上讲, 属于蒙特卡洛方法, 有很好的数学特性, 收玫于全局最优解。但是收玫速度慢, 执行时间长，算法性能与初始值有关。改进的SA算法区分 了全局优化和局部优化的特性, 更利于两种优化过程的收 玫进行, 数值优化的实验证明了改进后的SA算法比传统算 法更稳定、更省事。

\section{致谢}

本文得到国家自然科学基金（N0.61305017）和江南 大学科学技术青年基金（NO. JUSRP11462）阶段性工作成 果之一。

\section{参考文献}

［1］李金旭, 黄悦悦, 朱广帧. 模拟退火算法及其改进算法的研 究 $[J]$. 计算机光盘软件与应用, 2014, 22:141-142。
[2] 阎庆. 新型遗传模拟退火算法求解物流配送路径问题 [J]. 计算机应用，2004，24(1)：261-263。

[3] 吴意乐, 何庆. 基于改进遗传模拟退火算法的WSN路径优化 算法 $[J]$. 计算机应用研究, 2016, 10:1-6。

［4］杨卫波, 王万良, 张景玲, 赵燕伟. 基于遗传模拟退火算法的 矩形件优化排样 [J]. 计算机工程与应用, 2016, 1-6。

[5] Kirkpatrick S, Jr Gelatt C D, Vecchi M P. Optimization by simulated annealing [J]. Science, 1983, $220(11)$ : 650-671.

[6] 蒋龙聪, 刘江平. 模拟退火算法及其改进 $[J]$. 工程地球物 理学报, 2007, 02:135-140。

[7] 梁旭. 现代智能优化混合算法及其应用 $[\mathrm{M}]$. 北京: 电子工业 出版社, 2011. 10。

[8] 史峰. MATLAB智能算法30个案例分析 [M]. 北京: 北京航空航 天大学出版社, 2011.7。

[9] 李香平. 模拟退火算法原理及改进 $[J]$. 软件导刊, 2008, $7(4)$ : $47-48$ 。

[10] 刘怀亮. 模拟退火算法及其改进 [J]. 广州大学学报, 2004, 4 (3) : 503-506。

[11] Bouleimen K, Lecocq H.. A new efficient simulated annealing algorithm for the resource-constrained project scheduling problem and its multiple mode version[J]. European Journal of Operational Research, 2003, $149(2): 268-281$.

[12] Michalewicz Z. 演化程序一遗传算法和数据编码的结合 $[M]$. 北京: 科学出版社, 2000。

[13] 程博, 杨育, 刘爱军, 陈伟, 邢青松. 基于遗传模拟退火算法 的大件公路运输路径选择优化 [J]. 计算机集成制造系 统, 2013, 04:879-887。

[14] 袁澎, 艾芉, 赵媛媛. 基于改进的遗传 - 模拟退火算法和误 差度分析原理的PMU多目标优化配置 [J]. 中国电机工程学 报, 2014, 13:2178-2187。

[15] Yun, H. A. Modified Cobb-Douglas production function model and its application[J]. IMA Journal of Management Mathematics, 2013, 10(2): 1-13. 